\title{
Impact of a change in physician reimbursement on bone mineral density testing in Ontario, Canada: a population-based study
}

\author{
Susan Jaglal PhD, Gillian Hawker MD MSc, Ruth Croxford MSc, Cathy Cameron MHSc, \\ Anne-Marie Schott MD PhD, Sarah Munce MSc, Sonya Allin PhD
}

\section{Abstract}

Background: On Apr. 1, 2008, a revision was made to the fee schedule for bone mineral density testing with dual-energy $x$-ray absorptiometry (DXA) in the province of Ontario, Canada, reducing the frequency of repeat screening in individuals at low risk of osteoporosis. We evaluated whether the change in physician reimbursement successfully promoted appropriate bone mineral density testing, with reduced use among women at low risk and increased use among women and men at higher risk of osteoporosis-related fracture.

Methods: We analyzed data from administrative databases on physician billings, hospital discharges and emergency department visits. We included all physician claims for DXA in the province to assess patterns in bone mineral density testing from Apr. 1, 2002, to Mar. 31, 2011. People at risk of an osteoporosis-related fracture were defined as women and men aged 65 years or more and those who had a recent $(<6 \mathrm{mo})$ fracture after age 40 years. Joinpoint regression analysis was used to examine trends in DXA testing.

Results: Before the policy change, the overall number of DXA tests increased from 433419 in 2002/03 to 507 658 in 2007/08; after revision of the fee schedule, the number decreased to 422915 by 2010/11. Most of this reduction was due to a decrease in the agestandardized rate of DXA testing among women deemed to be at low risk, from 5.7 per 100 population in 2008/09 to 1.8 per 100 in 2010/11. In the high-risk group of people aged 65 or more, the age-standardized rate of testing increased after the policy change among men but decreased among women. Among those at high risk because of a recent clinical fracture, the age-standardized rate of DXA testing increased for both sexes and then decreased after the policy change.

Interpretation: A change in reimbursement designed to restrict access to bone mineral density testing among low-risk women was associated with an overall reduction in testing. Efforts to communicate guidelines for bone mineral density testing with greater clarity, particularly as they relate to high-risk individuals, need to be explored.

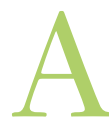

bone mineral density test using dual-energy $\mathrm{x}$-ray absorptiometry (DXA) evaluates the quantity of bone mineral and is used to make a diagnosis of reduced bone mass or osteoporosis and to provide information that contributes to an assessment of fracture risk. Together with other risk factors for fracture, information gained from a bone mineral density test can guide clinicians and patients in understanding the risk of an osteoporosis-related fracture; it can also inform decisions aimed at mitigating these risks (e.g., initiation of bisphosphonates treatment)., ${ }^{1,2}$

Clinical practice guidelines in Canada currently recommend bone mineral density testing in at-risk populations, namely all men and women aged 65 years or more and those who have had a fragility fracture after age 40 years. ${ }^{2,3}$ The recommended management model is based on assessment of fracture risk, which is derived in part from measured bone mineral density and appears on bone mineral density reports for most patients over age 50. For patients assessed as high risk, guidelines indi- cate that there is good evidence to support pharmacotherapy; for those assessed as low risk, guidelines state that patients are unlikely to benefit from pharmacotherapy and should be reassessed in 5 years. ${ }^{3}$ Thus, bone mineral density testing, as well as knowledge of clinical risk factors that can modify the assessment of fracture risk, are important components of prevention efforts to reduce (secondary) fracture risk., ${ }^{1,3}$

In the province of Ontario, Canada, a sharp increase in the rate of bone mineral density testing among women aged 4044 years, for whom fracture risk is typically low, was observed

Competing interests: None declared.

This article has been peer reviewed.

Correspondence to: Susan Jaglal, susan.jaglal@utoronto.ca CMAJ Open 2014.DOI:10.9778/cmajo.20130052 
in 2002, before major restrictions to testing were implemented. ${ }^{4}$ In fiscal year 2007/08, testing among women aged 40-59 years accounted for almost half (about 200000 ) of all bone mineral density tests performed. ${ }^{5}$ Although this high rate may indicate concern about osteoporosis as women approach menopause, it also suggests unnecessary testing. In an effort to curb testing among low-risk patients, on Apr. 1, 2008, the fee schedule was changed to limit DXA testing in low-risk patients to once every 36 months. Previously (from Oct. 1, 1999, to Mar. 31, 2008), DXA testing in such patients was allowed once every 24 months. In addition, a new fee code for a "baseline" test was added, and patients were limited to 1 baseline test in their lifetime. ${ }^{6}$ Both before and after 2008, high-risk individuals were allowed to undergo DXA testing annually. Therefore, the practice of referring low- but not high-risk patients was subject to modification.

We conducted a study to determine whether the change in the fee schedule successfully promoted appropriate bone mineral density testing, with reduced use among women at low risk and increased use among women and men at higher risk of osteoporosis-related fracture. Specifically, we examined trends in rates of DXA testing overall and in 2 groups: women at low risk, and women and men at risk of fracture due to advanced age or a previous osteoporosis-related fracture.

\section{Methods}

The study design was approved by the Research Ethics Board of the Sunnybrook Health Sciences Centre, University of Toronto.

\section{Data sources and study populations}

We identified DXA tests from the Ontario Health Insurance Plan (OHIP) claims database, which contains all physician fee-for-service billings in the province. ${ }^{7,8}$ In recent work to relate OHIP billings for DXA tests to DXA tests captured in electronic medical records of Ontario's family health teams, at least $92 \%$ of the tests in the electronic medical records were found to have been billed to the province. ${ }^{9}$ The OHIP billing database therefore provides a comprehensive representation of the province's testing patterns.

To obtain the age and sex of each DXA recipient, we linked the OHIP claims to the Registered Persons Database. Eligible claims included those for DXA tests performed in individuals aged 40 years and older between Apr. 1, 2002, and Mar. 31, 2011. The fee code in each claim allowed all patients to be classified as low or high risk for osteoporosis-related fracture. Tests performed after 2008/09 were also allowed to be categorized as baseline (i.e., distinct from the risk level code).

We identified 2 at-risk populations who, according to clinical practice guidelines, should receive DXA tests: women and men aged 65 or more who had no recent $(\leq 3 \mathrm{yr})$ DXA test and those who had a recent $(\leq 6 \mathrm{mo})$ fracture of the hip, wrist, spine, shoulder or pelvis. These types of fractures were selected because they are commonly associated with osteoporosis. Guidelines state that all individuals who have had a recent fracture should undergo testing to determine whether there is a need to start or change a medication regimen., ${ }^{1,3}$ The only exception to this rule is for individuals with bone mineral density tests on file within the year before a fracture, since bone mineral density is known not to change rapidly.,

We obtained data on clinical hip fractures from the Canadian Institute for Health Information hospital discharge abstract database, as described previously. ${ }^{8,10}$ We identified hip fractures in this database using the following International Statistical Classification of Diseases and Related Health Problems, 10th revision (ICD-10) codes: S72.0 ("fracture of neck of femur"), S72.1 ("pertrochanteric fracture") and S72.2 ("subtrochanteric fracture"). To identify clinical fractures of the wrist, spine, shoulder and pelvis, we obtained data from the National Ambulatory Care Resources database, which includes all emergency department visits, and the OHIP database. All patients who were admitted to hospital with a diagnosis of wrist fracture were identified; we then searched the National Ambulatory Care Resources database for patients with wrist fracture who were not admitted to hospital but were seen in the emergency department (ICD-10 code S52). Finally, to identify patients whose fracture was treated in a physician's office, we searched the OHIP database for incidents of 2 physician claims with a diagnosis of wrist fracture that were dated within 3 months of each other; the date of the fracture was defined by the patient's first visit. A similar procedure was followed for spine, shoulder and pelvis fractures, using the following ICD-10 codes: S22.0, S22.1, S32.0, S32.7, S32.8 (spine); S42.2 (shoulder); and S32.1, S32.3, S42.4, S32.5, S32.7, S32.8 (pelvis). We excluded patients if they had had a bone mineral density test during the 12 months before their fracture or if they died within 12 months after their fracture. Fractures due to multitrauma were excluded, because we included only cases of isolated fracture without other diagnostic codes. We used census population estimates supplied by Statistics Canada to compute age-standardized rates.

\section{Definition of fracture risk in the fee schedule}

Patients at high risk of an osteoporosis-related fracture may be assessed annually and are defined in the fee schedule as being at risk for accelerated bone loss, having osteopenia or osteoporosis identified on a previous DXA test or having bone loss in excess of $1 \%$ per year as shown by previous DXA testing. All other patients are defined as being at low risk of an osteoporosis-related fracture. The referring physician usually suggests the risk categorization upon ordering a DXA test; the risk category is often confirmed by the scanning facility before claim submission.

\section{Determining rates of DXA testing}

To examine time trends in DXA testing, we categorized physician claims for DXA tests by patient risk level (baseline, low risk, high risk) and directly standardized them based on census data.

To determine the proportion of patients who underwent bone mineral density testing after age 65 in each fiscal year, we selected records for patients aged 68-70 years who had had no DXA test between age 55 and 65. The proportion of 
individuals who received at least 1 DXA test in the 3-5 years after turning 65 was then calculated for each fiscal year. We restricted this indicator to a 3 -year period for each patient to exclude repeat tests labelled "low risk." As in the old fee schedule, the new schedule allows repeat testing after 3 years for low-risk patients.

We calculated rates of DXA testing following recent $(\leq 6 \mathrm{mo})$ clinical fracture by patient sex and type of fracture. We standardized the rates by age using census data.

\section{Statistical analysis}

We used joinpoint regression analysis to identify points where a statistically significant change in the linear slope of the trend line occurred over time, as described by Kim and colleagues. ${ }^{11}$ In the final model, each joinpoint indicates a statistically significant change in trend. An annual percentage change was computed for each trend by means of a generalized linear model that assumed a Poisson distribution. We performed joinpoint regression analyses using software from the Surveillance Research Program of the US National Cancer Institute (Joinpoint Regression Program, version 3.5, http://surveillance .cancer.gov/joinpoint/).

\section{Results}

\section{Rates of DXA testing}

The overall number of DXA tests increased until fiscal year 2007/08 (from 433419 in 2002/03 to 507658 in 2007/08) and then decreased to 422915 in 2010/11. Among women aged 40 years or more, the rate of DXA testing was fairly consistent between 2002/03 and 2006/07 (Figure 1). Beginning in $2008 / 09$, both the number and the rate in this group decreased. The values were lower in 2010/11 than in 2002/03 (no. of tests: 364874 v. 400 880; rate per 100: 10.4 v. 14.1 [Figure 1]). The rate of DXA testing among men aged 40 years or more increased gradually each year before it levelled off at about 2 per 100 in 2008/09 (Figure 1).
Table 1 shows the number and age-standardized rates of DXA tests among women, stratified by whether the patient was at low or high risk of fracture based on her test result. From $2002 / 03$ to $2007 / 08$, the rate did not change substantially. Beginning Apr. 1, 2008, rates among low-risk women decreased significantly. We therefore reject the null hypothesis of no joinpoint for women $(p=0.02)$. In contrast, the regression analysis for the low-risk and baseline tests among men showed no evidence of a joinpoint in the regression line $(p=0.4)$. The slope of the line for men was 0.061 (standard error $0.006, p<0.001$ ), which indicated that rates increased steadily among men.

\section{DXA testing among older patients}

Among older women, the age-standardized rate of DXA testing increased from 42.8 per 100 in $2002 / 03$ to 46.9 per 100 in 2005/06 and decreased each year thereafter. The largest drops were from $2007 / 08$ to $2008 / 09$ and from $2009 / 10$ to $2010 / 11$ (Figure 2). In contrast, the age-standardized rate of testing

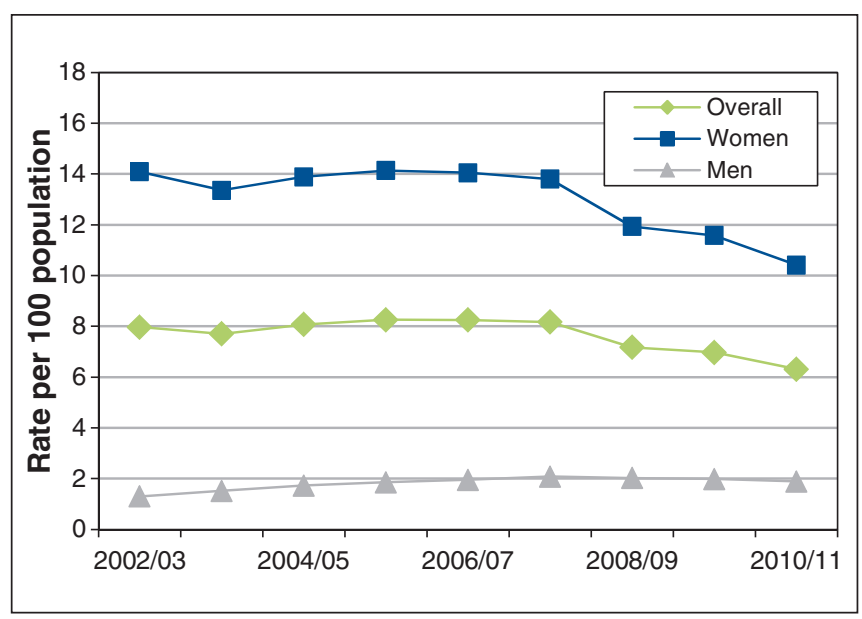

Figure 1: Age-standardized rates of dual-energy x-ray absorptiometry among women and men aged 40 years or more, 2002/03 to 2010/11.

\begin{tabular}{|c|c|c|c|c|c|c|}
\hline \multirow[b]{2}{*}{ Year } & \multicolumn{2}{|c|}{ Baseline* } & \multicolumn{2}{|c|}{ Low risk } & \multicolumn{2}{|c|}{ High risk } \\
\hline & No. & Rate per 100 & No. & Rate per 100 & No. & Rate per 100 \\
\hline $2002 / 03$ & - & - & 178325 & 6.3 & 222555 & 7.8 \\
\hline $2003 / 04$ & - & - & 161028 & 5.5 & 230408 & 7.8 \\
\hline $2004 / 05$ & - & - & 168277 & 5.6 & 249521 & 8.3 \\
\hline $2005 / 06$ & - & - & 167821 & 5.5 & 267710 & 8.7 \\
\hline $2006 / 07$ & - & - & 175988 & 5.6 & 269038 & 8.5 \\
\hline $2007 / 08$ & - & - & 184080 & 5.7 & 264427 & 8.1 \\
\hline $2008 / 09$ & 49800 & 1.5 & 101147 & 3.0 & 247400 & 7.4 \\
\hline $2009 / 10$ & 52349 & 1.6 & 95583 & 2.8 & 248793 & 7.2 \\
\hline $2010 / 11$ & 56145 & 1.6 & 62927 & 1.8 & 245802 & 7.0 \\
\hline
\end{tabular}




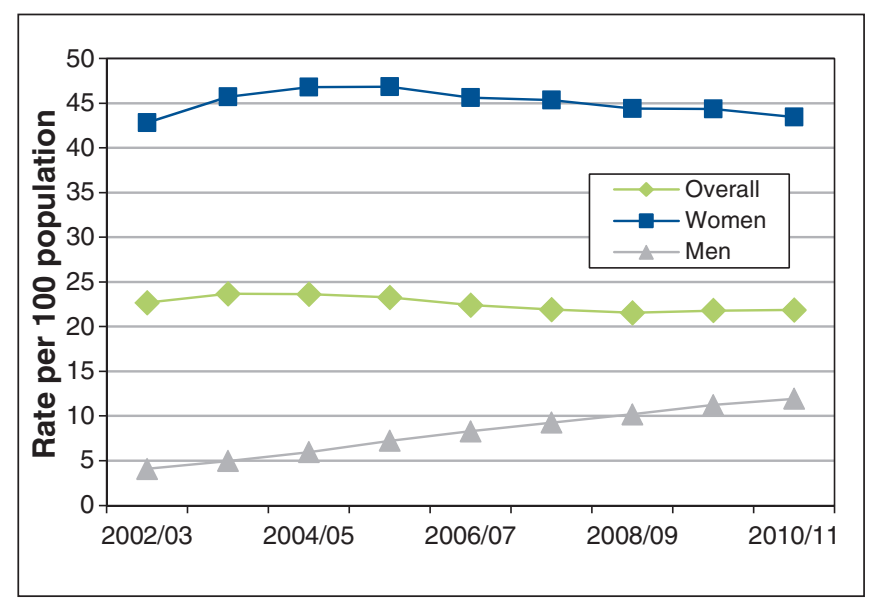

Figure 2: Age-standardized rates of dual-energy x-ray absorptiometry among women and men aged 68-70 years not previously tested, $2002 / 03$ to $2010 / 11$.

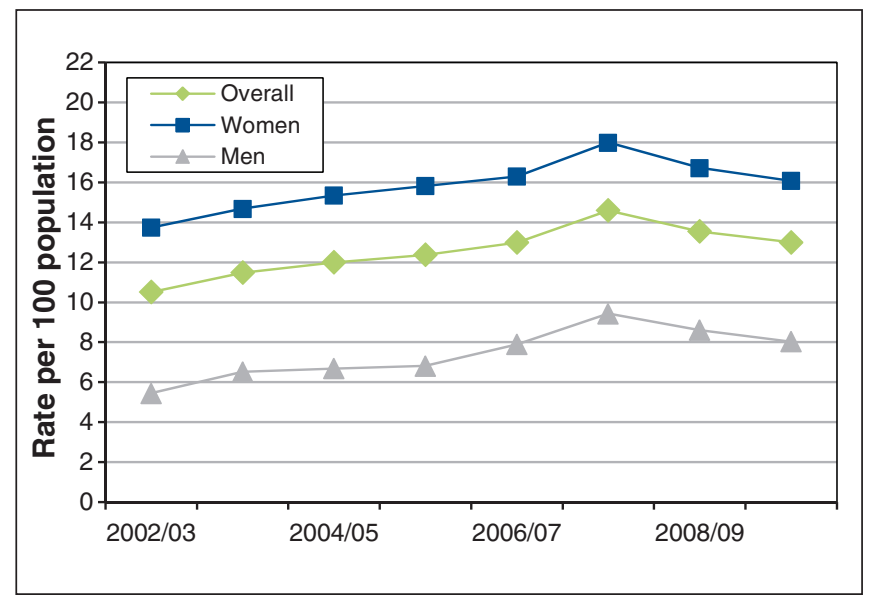

Figure 3: Age-standardized rates of dual-energy x-ray absorptiometry performed within 6 months after a fracture among women and men aged 40 years or more, 2002/03 to 2009/10.

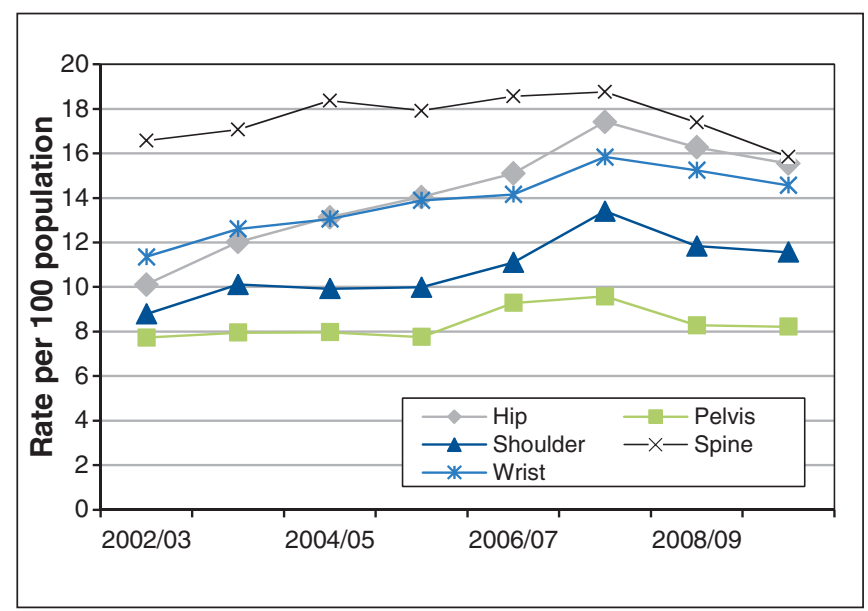

Figure 4: Age-standardized rates of dual-energy x-ray absorptiometry performed within 6 months after a fracture among adults aged 40 years or more, by fracture type, 2002/03 to 2009/10. among older men consistently increased from 2002/03 to 2010/11 (from 4.1 to 11.9 per 100 respectively) but remained well below the rate among older women (Figure 2).

\section{DXA testing following a recent clinical fracture}

Among patients with a clinical fracture of the hip, spine, wrist, shoulder or pelvis, rates of DXA testing within 6 months after fracture increased each year until 2007/08. During that time, the rates increased by 0.98 per 100 fractures for both sexes. After the revision in the fee schedule, the rates decreased and, by 2009/10, returned to 2006/07 levels (Figure 3). In 2009/10, the overall rate was 13.0 per 100 population (16.1 per 100 women and 8.0 per 100 men), as compared with 14.6 per 100 population (18.0 per 100 women and 9.4 per 100 men) in $2007 / 08$. Joinpoint regression analysis detected an increase in overall testing rates until 2007/08 and then a plateau in the rates.

Figure 4 shows the rate of DXA testing within 6 months after clinical fracture by fracture type. In 2009/10, the rate of testing after spinal fracture (15.9 per 100) or hip fracture (15.5 per 100) was higher than the rate after a wrist, pelvic or shoulder fracture. The rates differed by type of fracture until 2008/09, when they decreased across all fracture types. There was a marked increase in DXA testing among patients with hip and wrist fractures from 2002/03 to 2007/08. The increase was pronounced among those with pelvic and shoulder fractures between 2005/06 and 2007/08 and more gradual among patients with clinical spine fractures between 2002/03 and 2007/08. Among patients with hip fractures, the rate at which DXA testing increased from 2002/03 to 2007/08 was not statistically different from the rate at which it decreased afterward. Also, the rate of DXA testing following hip fracture was significantly higher among women than among men $(p<0.001)$.

\section{Interpretation}

Our results show that the change in physician reimbursement for bone mineral density testing in April 2008 was associated with a decrease in the overall rate of DXA testing in subsequent years, with a significant decrease among women aged 40 or more who were at low risk for an osteoporosis-related fracture. Among men aged 40 or more, the rate of DXA testing levelled off after 2008/09. Our findings suggest that the revised fee schedule may have reduced some unnecessary DXA testing among low-risk women.

There is documented inappropriate use of DXA testing in low-risk populations. Prior research has shown that, among 944 healthy women in Ontario referred for a baseline DXA test at midlife, more than $90 \%$ had normal bone density. ${ }^{12}$ Similarly, in a US cohort of 615 women aged 49 years or more who received a baseline DXA test, Schnatz and colleagues ${ }^{13}$ found that $40 \%$ did not meet the North American Menopause Society criteria for testing. ${ }^{2}$ In a recent study involving 4957 women aged 67 years or more with normal bone mineral density or osteopenia, osteoporosis was detected in less than $10 \%$ during rescreening intervals of about 15 years. ${ }^{14}$ 
Clinical guidelines indicate that women and men aged 65 or more should undergo bone mineral density testing. . $2,315-17^{15}$ However, in our study, the rate of DXA testing among eligible older women decreased after 2005/06; this decrease became slightly more pronounced after the revised fee schedule was introduced in 2008. In contrast, the rate of testing among older men increased each year, although it was well below the rate among older women. These findings suggest that guidelines for DXA testing among older women are not being followed. In 2010, only $43 \%$ of older women in Ontario who became eligible for a DXA were tested. In a recent survey by the Public Health Agency of Canada, only 47\% of Canadians aged 65 years or more reported ever having had a DXA test. ${ }^{18}$

When we turned our attention to bone mineral density testing among individuals with a recent history of fragility fracture, the effect of the revised fee schedule was pronounced. Before 2008, the rate of DXA testing within 6 months after a fragility fracture had been increasing annually. In 2008, the rate began to decrease, such that in 2009/10 it returned to 2006/07 levels. If this trend continues, we can expect to return to the 2002/03 rate for testing after hip fracture by the end of 2013/14. This indicates underuse of DXA testing among at least one high-risk group (patients with recent fractures) and suggests that the change in fee schedule may have compromised initiatives from the Ontario Osteoporosis Strategy, a population-based chronic disease management strategy designed to reduce fractures. ${ }^{19}$ Tools to help practitioners identify high-risk individuals, such as a standardized requisition for referral that clarifies clinical guidelines for bone mineral density testing, may help to ensure appropriate testing in the face of funding changes.

Our results highlight unintended consequences of a government policy intended to reduce inappropriate DXA testing among low-risk patients. The new reimbursement policy was associated with an overall reduction in testing and a sharp reduction in at least 1 high-risk population (patients with a recent fracture). Why the new policy was interpreted so broadly remains to be determined. One potential explanation is confusion among physicians. The policy dictated by the revised fee schedule is inconsistent with current clinical guidelines, still referring to guidelines from $2002 .{ }^{20}$ In addition, the fee schedule provides no guidance for referral of patients with a recent fracture or of patients 65 and older. It is unclear how physicians should arrive at decisions to refer given the competing information in guidelines and from OHIP. Another explanation may be changing patient perceptions, which may have coincidentally aligned with policy change. Patients may not want to consider taking osteoporosis medications ${ }^{21}$ or may be concerned about drug safety and effectiveness. ${ }^{22}$ As a result, they may see DXA testing as irrelevant. Alternatively, patients may lack understanding of the link between bone mineral density and fracture risk ${ }^{21}$ and therefore undervalue DXA testing.

Why the change in physician reimbursement was associated with such a dramatic decrease in the rate of DXA testing among patients with recent fracture is of particular concern. Additional research to understand the failure to enforce guidelines in this patient population is required. For a patient to undergo a bone mineral density test within 6 months after a fracture, he or she has to have been assessed by a primary care physician or been part of a systematic osteoporosis management program. Whether the policy change affected rates of referral within primary care more so than rates of referral within osteoporosis programs cannot be determined based on administrative data. We therefore do not yet know where best to direct additional efforts to promote guideline implementation in this high-risk group.

\section{Limitations}

Our study has limitations. First, the categorization of fracture risk as low or high was determined, for the most part, by the referring physicians; we were unable to verify the risk designations using the administrative data. This means an unknown amount of misclassification exists in our results. Some low-risk patients may have undergone testing after the policy change because they were coded as being at high risk. However, the overall rates of DXA testing declined measurably across the board after the policy change.

Second, we could not separate baseline from repeat DXA measurements before 2008, which made it difficult for us to separate patients being screened from those being monitored. We restricted our analysis to tests that appeared to be baseline tests by including only individuals aged 68-70 without a history of DXA testing after age 55.

Third, because of the lack of administrative data identifying pathways of care among patients with fractures, we could not determine accurately where more effort was needed to improve referral rates. This remains the subject of future research.

Fourth, we chose to define high-risk patients as individuals aged 65 or more and patients aged 40 or more with a recent fracture. We did this because these patients represent the majority of high-risk patients and are more likely to be seen in the primary care setting. Patients with glucocorticoid and rheumatoid arthritis represent about $5 \%$ of high-risk patients, and they tend to be younger.

We did not include a longer time horizon for previous or recurrent fracture because we hypothesized that, if an effect of less testing was observed among patients with a recent fracture, it might have been even lower among those with an older fracture. The intent was that the recent fracture should have prompted the bone mineral density test, which is the message promoted in the guidelines. If a recent fracture did not prompt the physician to order a bone mineral density test, a fracture that occurred more than a year earlier would be less likely to do so.

Finally, we do not yet know how changes in referral practices for bone mineral density testing will influence subsequent treatment and fracture rates. We need a much longer follow-up period to determine the impact on fracture rates. Instead, we relied on evidence that relates the occurrence of bone mineral density testing to better follow-up and improved treatment rates in order to justify our claim that reduced testing may compromise the management of patients, especially those at high risk. ${ }^{1,3,19}$ 


\section{Conclusion}

Our study suggests that a change in physician reimbursement to reduce bone mineral density testing among women at low risk had both a positive and a negative effect. The policy change was associated with an overall reduction in DXA testing. On the positive side, potentially unnecessary DXA testing in low-risk groups was reduced. On the negative side, DXA testing in high-risk groups was also reduced, which may further compromise the quality of osteoporosis care in Ontario.

How changes to policy have come to stand in the way of guideline implementation remains unclear. What is clear, however, is the inconsistency with which guidelines are followed at the point of referral. Efforts to communicate guidelines with greater clarity at this juncture, particularly as they relate to high-risk individuals, are worth exploration. A standardized requisition for referral that clarifies guidelines for bone mineral density testing to practitioners is one mechanism that may help to ensure appropriate testing in the face of funding changes.

\section{References}

1. Lentle B, Cheung AM, Hanley DA, et al. Osteoporosis Canada 2010 guidelines for the assessment of fracture risk. Can Assoc Radiol 7 2011;62:243-50.

2. North American Menopause Society. Management of osteoporosis in postmenopausal women: 2010 position statement of The North American Menopause Society. Menopause 2010;17:25-54.

3. Papaioannou A, Morin S, Cheung AM, et al. 2010 clinical practice guidelines for the diagnosis and management of osteoporosis in Canada: summary. CMAF 2010;182:1864-73.

4. Jaglal SB. Bone density testing. In: Stewart DE, Cheung AM, Ferris LE, et al., editors. Ontario Women's Health Status Report. Toronto (ON): Ontario Women's Health Council; 2002: 391.

5. Ontario Health Insurance Plan: database. Toronto (ON): Institute for Clinical and Evaluative Sciences; 2009.

6. Schedule of benefits changes - April 1, 2008. Toronto (ON): Ontario Ministry of Health and Long-Term Care; 2008. Available: www.health.gov.on.ca/english /providers/program/ohip/bulletins/4000/bul4470a.pdf (accessed 2012 Aug. 24).

7. Jaglal SB, McIsaac W, Hawker G, et al. Patterns of use of bone density testing in Ontario, 1992-1998. CMA7 2000;163:1139-43.

8. Jaglal SB, Weller I, Mamdami M. Population trends in bone density testing, treatment and hip and wrist fracture rates: Are the hip fracture projections wrong? 7 Bone Miner Res 2005;20:898-905.

9. Tu K, Green D, Young J, et al. Bone mineral density (BMD) database feasibility study. Toronto (ON): Institute for Clinical and Evaluative Sciences; 2012.

10. Leslie WD, O'Donnell S, Jean S, et al. Trends in hip fracture rates in Canada. ҰAMA 2009;302:883-9.

11. Kim HJ, Fay MP, Feuer EJ, et al. Permutation tests for JoinPoint regression with applications to cancer rates. Stat Med 2000;19:335-51.

12. Hawker G, Mendel A, Lam MA, et al. A clinical decision rule to enhance targeted bone mineral density testing in healthy mid-life women. Osteoporos Int 2012; 23:1931-8.

13. Schnatz PF, Marakovits KA, DuBois M, et al. Osteoporosis screening and treatment guidelines: Are they being followed? Menopause 2011;18:1072-8.

14. Gourlay ML, Fine JP, Preisser JS, et al. Bone-density testing interval and transition to osteoporosis in older women. NEngl F Med 2012;366:225-33.
15. Physician's guide to prevention and treatment of osteoporosis. Washington: National Osteoporosis Foundation; 1998:16, 20, 22.

16. Nordin C. Screening for osteoporosis: U.S. Preventive Services Task Force recommendation statement. Ann Intern Med 2011;155:276; author reply 276-7.

17. Writing Group for the ISCD Position Development Conference. Indications and reporting for dual x-ray absorptiometry. 7 Clin Densitom 2004;7:37-44.

18. What is the impact of osteoporosis in Canada and what are Canadians doing to maintain bealthy bones? Ottawa (ON): Public Health Agency of Canada; 2010. Available: www.phac-aspc.gc.ca/cd-mc/osteoporosis-osteoporose/index-eng.php (accessed 2012 Aug. 24).

19. Jaglal SB, Hawker GA, Cameron C, et al. The Ontario Osteoporosis Strategy: implementation of a population-based osteoporosis action plan in Canada. Osteoporos Int 2010;21:903-8.

20. Brown JP, Josse RG; Scientific Advisory Council of the Osteoporosis Society of Canada. 2002 clinical practice guidelines for the diagnosis and management of osteoporosis in Canada. CMA7 2002;167(10 Suppl):S1-34.

21. Sale JE, Gignac MA, Hawker G, et al. Decision to take osteoporosis medication in patients who have had a fracture and are 'high' risk for future fracture: a qualitative study. BMC Musculoskelet Disord 2011;12:92.

22. Sambrook PN, Chen JS, Simpson JM, et al. Impact of adverse news media on prescriptions for osteoporosis: effect on fractures and mortality. Med 7 Aust 2010;193:154-6.

Affiliations: Departments of Physical Therapy (Jaglal, Cameron, Allin) and Medicine (Hawker), University of Toronto; Institute for Clinical Evaluative Sciences (Jaglal, Croxford); Institute of Health, Policy, Management and Evaluation (Hawker, Munce), University of Toronto; the Women's College Research Institute (Jaglal, Hawker), Toronto, Ont.; Hospices Civils de Lyon (Schott), Lyon, France; and Toronto Rehabilitation Institute (Allin), University Health Network, Toronto, Ont.

Contributors: Susan Jaglal, Ruth Croxford and Cathy Cameron were responsible for the conception and design of the study and the acquisition and analysis of the data. Susan Jaglal drafted the manuscript. All of the authors contributed substantially to the interpretation of the data, revised it critically for important intellectual content, approved the final version submitted for publication and agree to act as guarantors of the work.

Acknowledgements: This study was supported by the Ontario Osteoporosis Strategy and the Institute for Clinical and Evaluative Sciences. Susan Jaglal holds and has support from the Toronto Rehabilitation Institute - University Health Network Chair at the University of Toronto in Health Services Research. Gillian Hawker is the physicianin-chief of the Department of Medicine at Women's College Hospital, where she holds and has support from the F.M. Hill Chair in Academic Women's Medicine. Sarah Munce was supported by a Frederick Banting and Charles Best Doctoral Research Award from the Canadian Institutes of Health Research and is currently supported by a Knowledge Translation Canada Fellowship Award.

Supplemental information: For reviewer comments and the original submission of this manuscript, please see www.cmajopen.ca/content /2/2/E45/suppl/DC1

Disclaimer: This study was supported in part by the Institute for Clinical Evaluative Sciences, a nonprofit research institute funded by the Ontario Ministry of Health and Long-Term Care (MOHLTC). The opinions, results and conclusions reported in this paper are those of the authors and are independent from the funding sources. No endorsement by ICES or the MOHLTC is intended or should be inferred. 